\title{
El cuerpo humano como sistema de aprovechamiento de la energía solar
}

\author{
Lina M. Escobar-Durangoํㅜ ; Ariel R. Becerra-Becerra² ${ }^{\text {; Claudia P. Parra- Medina }}{ }^{3}$ \\ ${ }^{1}$ Grupo de Investigación Integrar, Departamento de Medicina, Universidad de Pamplona, Colombia \\ ${ }^{2}$ Grupo de Investigación Integrar, Departamento de Física y Geología, Universidad de Pamplona, \\ Colombia \\ ${ }^{3}$ Grupo de Investigación Integrar, Departamento de Ingeniería Mecánica, Universidad de Pamplona, \\ Colombia
}

\section{Resumen}

Este artículo presenta una caracterización del comportamiento del cuerpo humano como un sistema complejo de aprovechamiento de la energía solar.

La mayor parte de los seres vivos tiene como su principal fuente de energía el Sol, sea esta fuente directa o indirecta, tal como sucede con los organismos autótrofos y heterótrofos dentro de los que podemos nombrar otras clasificaciones como fotótrofos, fotoautótrofos, quimioautótrofos y otros. Se resalta que los organismos heterótrofos en parte toman su energía de los autótrofos, y para estos últimos la mayor parte de energía proviene del Sol. En el presente trabajo se analiza el ser humano como un organismo dependiente en gran parte de la energía solar aunque un tanto por ciento de esa energía proviene indirectamente. Se hace una clasificación de los subsistemas de aprovechamiento en el hombre y se demuestra que si tenemos en cuenta hasta una tercera etapa de la cadena alimenticia el hombre como muchos organismos, es dependiente en un gran porcentaje de la energía suministrada por el Sol.

Palabras Clave: Aprovechamiento, energía solar, fuentes de energía, ser humano.

\section{The human body as a system of utilization of solar energy}

\section{Abstract}

This article presents a characterization of the behavior of the human body like a complex system of use of the solar energy.

Bistua Revista de la Facultad de Ciencias Basicas .Universidad de Pamplona. ISSN 0120-4211 
The majority of living being have like a principal source of energy the sun, been this a direct or indirect sources, like had succeed with the autótrofos and heterótrofos organisms inside which we can mention other classification such as fototrofos, fotoautotrofos, quimioautotrofos and others.

It is highlighted that the heterótrofos organisms take its energy of the autótrofos, and for the above mentioned energy come from the sun. In the present work it is analyzed the human being as a dependent organisms largely on the solar energy although some porcent from this energy it comes indirectly. It do a classification of the food that the man consumes in accordance with its energy main source and it is demonstrated that if we have in mind to a third stage of nutritive chain the man like many organisms, is dependent of the energy given by the sun.

Keywords: Use, solar energy, energy sources, human body.

*Para citar este artículo: Escobar-Durango LM; Becerra-Becerra AR; Parra- Medina CP. El cuerpo humano como sistema de aprovechamiento de la energía solar.Revista Bistua.2016.14(1):91-99

+ Autor para el envió de correspondencia y la solicitud de las separatas: Escobar-Durango LM. ${ }^{1}$ Grupo de Investigación Integrar, Departamento de Medicina, Universidad de Pamplona, Colombia.. email: Imescobar@gmail.com. 
93

\section{Introducción}

A lo largo de la historia, la especie humana ha tenido una relación especial con el Sol. De hecho, no hay ningún aspecto en el entorno que no esté influenciado por el Sol. Desde la fotosíntesis hasta el estado de ánimo propio, están condicionados por dicho astro. No es de extrañar, pues, el papel central que juega la radiación solar en nuestra vida diaria.

La energía es el combustible que el cuerpo humano necesita para vivir y ser productivo. Todos los procesos que se realizan en las células y en los tejidos producen y requieren energía para llevarse a cabo. Todos los seres vivos requieren energía para realizar los procesos vitales, como la nutrición, el movimiento y la reproducción. De forma particular, el ser humano utiliza la energía para muchos propósitos como: caminar, correr, moverse, respirar, crecer, madurar los tejidos, producir leche materna y mantener los tejidos sanos, entre otros. La energía necesaria para vivir se obtiene de los alimentos, los cuales a su vez se sintetizan en su origen aprovechando directa o indirectamente la energía del Sol. En términos generales, las necesidades de energía estarán satisfechas cuando el consumo de alimentos es el adecuado para mantener un buen desarrollo del cuerpo. De una u otra manera esa interacción con la energía proveniente del Sol se define por el aprovechamiento directo (exposición a la radiación) 0 indirecto (energía tomada de los alimentos) que los seres humanos realizan. En términos generales, la energía directa es muy poca y está relacionada principalmente con la radiación directa que absorbe el organismo a través de la piel y otros transductores biológicos. Por otra parte, la energía que se puede aprovechar de manera indirecta es mayoritaria y se determina por el metabolismo energético de los seres humanos, es decir, el conjunto de procesos físicos y químicos del cuerpo que convierten o usan energía, tales como: la respiración, la circulación sanguínea, la regulación de la temperatura corporal, la contracción muscular, la digestión de alimentos y nutrientes, la eliminación de los desechos a través de la orina y de las heces, el funcionamiento del cerebro y los nervios ; por lo tanto, es de suma importancia conocer estos procedimientos, porque juegan un papel fundamental en la regulación y utilización de la energía en el interior del cuerpo humano.

En el presente artículo se analizarán los subsistemas componentes del sistema de aprovechamiento del organismo humano y su analogía con los sistemas convencionales de aprovechamiento de la energía solar.

\section{Desarrollo Teórico}

Es posible entonces en relación al análisis de los subsistemas componentes del aprovechamiento de la energía solar y su función realizar una comparación respecto a los elementos de los sistemas tecnológicos y del organismo humano. En la tabla 1 se presenta un cuadro comparativo de las funciones 
análogas de la caracterización de los sistemas de aprovechamiento de la energía solar. Básicamente se identifican en primera instancia los captadores de energía del cuerpo humano como la piel ${ }^{1}$, el estómago e intestinos ${ }^{2}$ y los pulmones ${ }^{3}$. La primera capta la radiación directa del Sol y la radiación en forma de calor, el segundo aprovecha la energía química (inicialmente solar) contenida en los alimentos, y finalmente los pulmones captan las moléculas de oxígeno que al fin y al cabo liberan energía en los procesos de oxidación. El transporte interno es realizado por el sistema circulatorio que es el encargado de transportar el oxígeno a los órganos y tejidos para realizar la oxidación.

El subsistema de regulación lo realizan los órganos que controlan el uso de la energía en el organismo humano que son el cerebro que es un órgano sumamente importante en el organismo, es denominado glucodependiente y por ello requiere de grandes cantidades de energía que le suministra la glucosa o en su defecto los cuerpos cetónicos para poder funcionar. El cerebro es el motor de procesamiento que tiene el cuerpo por excelencia ${ }^{4}$, y el hígado, una de sus funciones es intervenir en el metabolismo de las grasas, sintetizar ácidos grasos, a partir de los aminoácidos y los azúcares, también produce colesterol, fosfolípidos y lipoproteínas, y oxida a las grasas para producir energía. La glucosa que no es utilizada por el organismo pasa al hígado para ser almacenada. ${ }^{5} \mathrm{El}$
94

sistema sensorial está determinado por los receptores sensoriales que son terminaciones nerviosas especializadas, ubicadas en los órganos sensoriales (como la lengua, la piel, la nariz, los ojos, el oído, entre otros). Son los encargados de recibir el estímulo para llevar la información sensorial para producir una respuesta ya sea interna o externa, capaces de captar estímulos internos o externos y generar un impulso nervioso y sensaciones $^{6}$. Por otra parte la energía que se consume en forma de alimentos 0 bebidas puede ser almacenada en el organismo en forma de grasa (tejido adiposo que constituye la mayor reserva energética), glucógeno (energía a corto plazo disponible para la producción de ATP), o proteínas, esta reserva energética es usada por el organismo en situaciones que lo requieran para solventar las funciones del mismo ${ }^{7}$. Finalmente el cuerpo humano aprovecha la energía generalmente en la realización de trabajo mecánico que constituye un trabajo útil para el organismo y en todos los procesos que comprenden el metabolismo energético ${ }^{8}$.

La eficiencia depende en un gran porcentaje de la realización de trabajo mecánico, por ejemplo de la energía libre de la hidrólisis del ATP que es aprovechada por el mecanismo contráctil de los músculos con una eficiencia del orden del $90 \%$ pero durante la contracción se producen otros procesos, como el 
transporte activo de calcio por el retículo sarcoplásmico, que ocurren con una eficiencia de sólo el $50 \%$ y que no se traducen precisamente en trabajo mecánico ${ }^{9}$.

\section{Resultados y Análisis}

La Figura 1 muestra una representación del esquema de los niveles de dependencia de los seres vivos del entorno solar. Existen en la naturaleza organismos dependientes de la energía del Sol. Dentro de la clasificación de los organismos por su dependencia energética es posible encontrar diferentes niveles en virtud de los procesos de recepción directa o mecanismos indirectos de aprovechamiento de la energía solar. Los organismos autótrofos son productores, es decir, se alimentan por sí mismos debido a que sintetizan sustancias para mutualismos a partir de otras sustancias inorgánicas. Esto significa que no necesitan de otros seres vivos para procesar los alimentos, sino que usan como única fuente la energía proveniente del Sol, usando la radiación, dióxido de carbono y sustancias químicas inorgánicas para la producción de azucares como el almidón y la glucosa, a este proceso se le denomina fotosíntesis. Los fotosintetizadores no son los únicos autótrofos, también hay seres vivos quimiosintetizadores que degradan elementos químicos para obtener energía (sustituyendo la de la luz) y realizar quimiosíntesis (otra forma de autotrofismo) ${ }^{10}$.
95

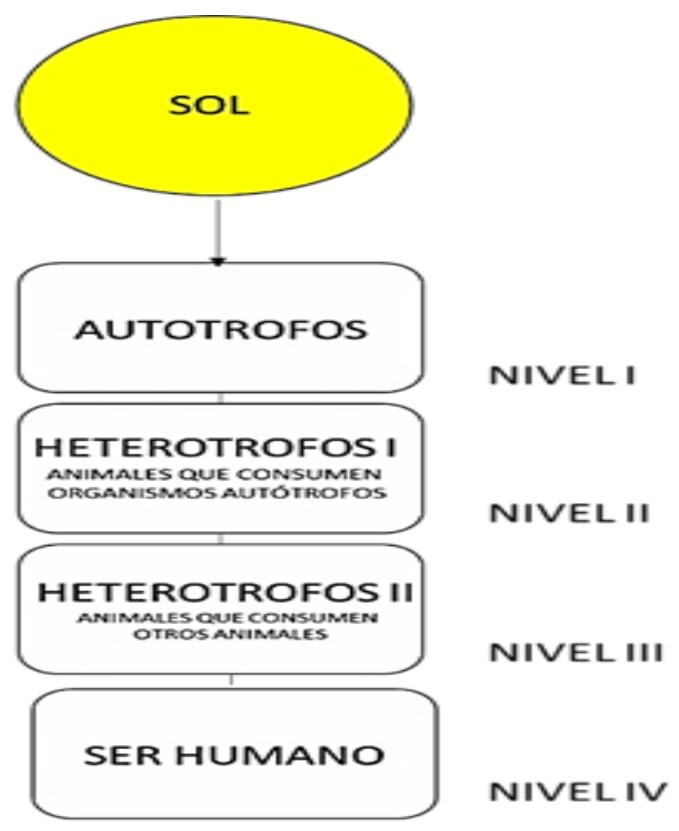

Figura 1. Niveles de dependencia del entorno solar

En las Figuras 2 y 3 se muestra el flujo del aprovechamiento de energía solar a partir de los procesos del organismo humano y los sistemas tecnológicos, respectivamente. Es importante hacer alusión a que existe una gran similitud en la serie de procesos de aprovechamiento de la energía en el organismo humano y los elementos constitutivos del mismo y los sistemas tecnológicos de energía solar, esto debido a que la configuración del sistema ser humano como una máquina lo estructuran en un centro de operaciones muy complejo, que debe mantenerse dentro de ciertos límites que le permitan desarrollar su funcionabilidad de manera óptima, mediante los procesos físicos y químicos que lleva a cabo y de los cuales la termodinámica representa una parte crucial del mismo, ya que 
son los procesos de regulación, transformación y producción de energía los que le permiten llevar a cabo todas sus funciones vitales.Los procesos tienen características físicas similares en los subsistemas de captación, almacenamiento y aprovechamiento de la energía solar, y es pertinente e importante reconocer que a partir de estos procesos que ocurren en la interacción directa o indirecta con la energía solar surgen las transformaciones energéticas requeridas por dichos sistemas. Por ejemplo en la captación los componentes de cada sistema tienen fundamentalmente una interacción directa. En el caso del ser humano la primera interacción es con la piel. Pese a que no existe mayor aprovechamiento en esta fase la posibilidad de captar la energía le permite al hombre la fijación de la vitamina $D$ en los huesos gracias a la absorción de calcio obtenido indirectamente por los alimentos. También es recibida la forma más común de energía, el calor. Este ayuda al ser humano a mantener la temperatura corporal constante. EI sistema térmico, por otra parte, puede a partir de la interacción directa absorber el calor producido por los rayos solares para la producción de agua caliente o calefacción. Otro de los elementos más interesantes del análisis es la transformación de la energía solar en energía química almacenada en las baterías de los sistemas fotovoltaicos. En el caso del organismo humano estos procesos suceden a través del ATP. Es posible Bistua Revista de la Facultad de Ciencias Basicas .Unive
96

apreciar que la energía es empleada por cada sistema particularmente en la realización de trabajo útil (químico, eléctrico, de transporte y mecánico) o su transformación en calor llevando a cabo reacciones de diferente tipo pero con procesos globalmente similares entre un sistema y otro.

\section{Conclusiones}

Una vez descritos los aspectos generales del organismo humano como sistema de energía solar se analizaron los diferentes órganos y componentes como sistema energético y abordamos el tema concreto del análisis comparativo con un sistema convencional de energía solar y los diferentes niveles de dependencia de esta. Fue posible determinar que el organismo humano, en su proceso de aprovechamiento de la energía es comparable con un sistema tecnológico de energía solar y se puede encontrar similitud en sus subsistemas: captación de la energía, transporte, regulación, almacenamiento y aprovechamiento. Algunos procesos en los dos sistemas comparados son muy similares como por ejemplo el almacenamiento, en donde la energía solar es almacenada en forma de energía química.

\section{Agradecimientos}

Los autores del presente artículo desean expresar su agradecimiento al grupo de investigación INTEGRAR de la Universidad de Pamplona y a la Vicerrectoría de Investigaciones, 
tanto por el apoyo económico para la divulgación del trabajo en eventos nacionales e internacionales, como por el apoyo humano para la publicación del presente artículo.

\section{Referencias bibliográficas}

[1] J. A. Carrion, «DMedicina.com,» Junio 2015. http://www.dmedicina.com/vidasana/actualidad/efectos-biologicos-delsol.

[2] «Fotobiologia y dermatologia,» Marzo 2012. [En línea]. Available: http://www.fyboa.uma.es/wpcontent/uploads/2012/03/-copia.pdf.J.-Y.

[3] A. S. Frumento, Biofísica, Buenos Aires: Mosby - Doyma Libros, 1995.

[4] «Sistema Regulador del cuerpo humano» [En línea]. Available: a.http://www.cyd.conacyt.gob.mx/237/Art iculos/Cuando_el_calor/Cuando_el_calor 2.html

[5] «Almacenadores de Energía,» [En línea]. Available:

http://es.265health.com/dietnutrition/healt hydiets/1008151129.html\#.VErKCvmUe E5.

[6] «Receptores de Calor Corporal, » Agosto 2014. [En línea]. Available: http://es.wikipedia.org/wiki/\%C3\%93rgan o sensorial\#Receptores.

[7] M. V. Volkenshtein, «Cuestiones y Métodos de la Biofísica,» de Biofísica, Mir, 1985, pp. 16-32.

[8] L. E., Bioquímica: Rutas metabólicas y transferencia de energía, Ediciones Omega, 2003.

[9] K. I. Some, Principles of energetics in biochemical reactions, Nueva York: Academic Press Inc, 1957.

[10] A. T., Biología, la vida en la Tierra, Pearson Education, 2003.

\begin{tabular}{|c|c|c|c|}
\hline $\begin{array}{c}\text { FUNCIÓ } \\
\text { N }\end{array}$ & $\begin{array}{l}\text { ORGANISMO } \\
\text { HUMANO }\end{array}$ & $\begin{array}{c}\text { SISTEMA } \\
\text { DE } \\
\text { ENERGÍA } \\
\text { SOLAR } \\
\text { TÉRMICA }\end{array}$ & $\begin{array}{c}\text { SISTEMA DE ENERGÍA } \\
\text { SOLAR } \\
\text { FOTOVOLTAICA }\end{array}$ \\
\hline $\begin{array}{l}\text { Captaci } \\
\text { ón }\end{array}$ & $\begin{array}{l}\text { Piel, } \\
\text { estomago, } \\
\text { pulmones }\end{array}$ & $\begin{array}{l}\text { Colectore } \\
\text { s solares }\end{array}$ & Paneles \\
\hline $\begin{array}{l}\text { Transp } \\
\text { orte } \\
\text { interno }\end{array}$ & $\begin{array}{l}\text { Sistema de } \\
\text { circulación }\end{array}$ & $\begin{array}{l}\text { Agua, } \\
\text { fluidos } \\
\text { termoesta } \\
\text { bles, } \\
\text { entre } \\
\text { otros }\end{array}$ & Cableado \\
\hline $\begin{array}{l}\text { Regula } \\
\text { ción }\end{array}$ & $\begin{array}{l}\text { Cerebro, } \\
\text { hígado }\end{array}$ & $\begin{array}{c}\text { Estación } \\
\text { de } \\
\text { control }\end{array}$ & Regulador, inversor \\
\hline $\begin{array}{l}\text { Sistema } \\
\text { Sensori } \\
\text { al }\end{array}$ & $\begin{array}{c}\text { Receptores, } \\
\text { electrorrecepto } \\
\text { res, } \\
\text { mecanorrecept } \\
\text { ores, } \\
\text { termorreceptor } \\
\text { es, } \\
\text { quimiorrecept } \\
\text { ores, } \\
\text { fotorreceptores }\end{array}$ & $\begin{array}{c}\text { Termocu } \\
\text { plas, } \\
\text { medidore } \\
\text { s de } \\
\text { presión y } \\
\text { de flujo, } \\
\text { sensor de } \\
\text { radiación }\end{array}$ & $\begin{array}{l}\text { Sensor de radiación, } \\
\text { temperatura, carga }\end{array}$ \\
\hline $\begin{array}{l}\text { Almace } \\
\text { namien } \\
\text { to }\end{array}$ & $\begin{array}{l}\text { ATP, Glucosa, } \\
\text { tejido adiposo, } \\
\text { otros tejidos }\end{array}$ & $\begin{array}{c}\text { Materiale } \\
\text { s de } \\
\text { cambio } \\
\text { de fase, } \\
\text { agua, } \\
\text { fluidos }\end{array}$ & $\begin{array}{c}\text { Baterías, rotores, aire } \\
\text { comprimido, energía } \\
\text { química, otros }\end{array}$ \\
\hline $\begin{array}{l}\text { Aprove } \\
\text { chamie } \\
\text { nto y } \\
\text { eficienc } \\
\text { ia }\end{array}$ & $\begin{array}{l}\text { Metabolismo } \\
\text { energético } \\
\text { Contracción } \\
\text { muscular }\end{array}$ & $\begin{array}{c}\text { Liberació } \\
\text { n de } \\
\text { energía } \\
\text { externa } \\
\text { para } \\
\text { diferentes } \\
\text { usos }\end{array}$ & $\begin{array}{c}\text { Liberación de energía } \\
\text { eléctrica para fines } \\
\text { externos }\end{array}$ \\
\hline
\end{tabular}

TABLA 1. Cuadro comparativo de las funciones análogas delos sistemas de energía solar tecnológicos y el ser humano 


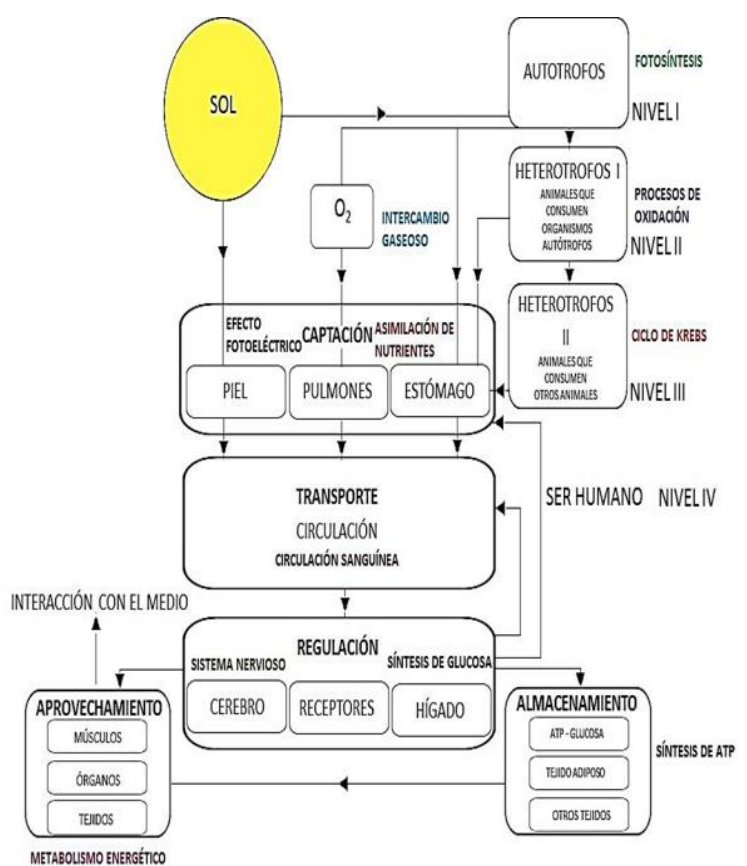

FIGURA 2. Diagrama de flujo de aprovechamiento de la energía solar por el organismo humanoprocesos

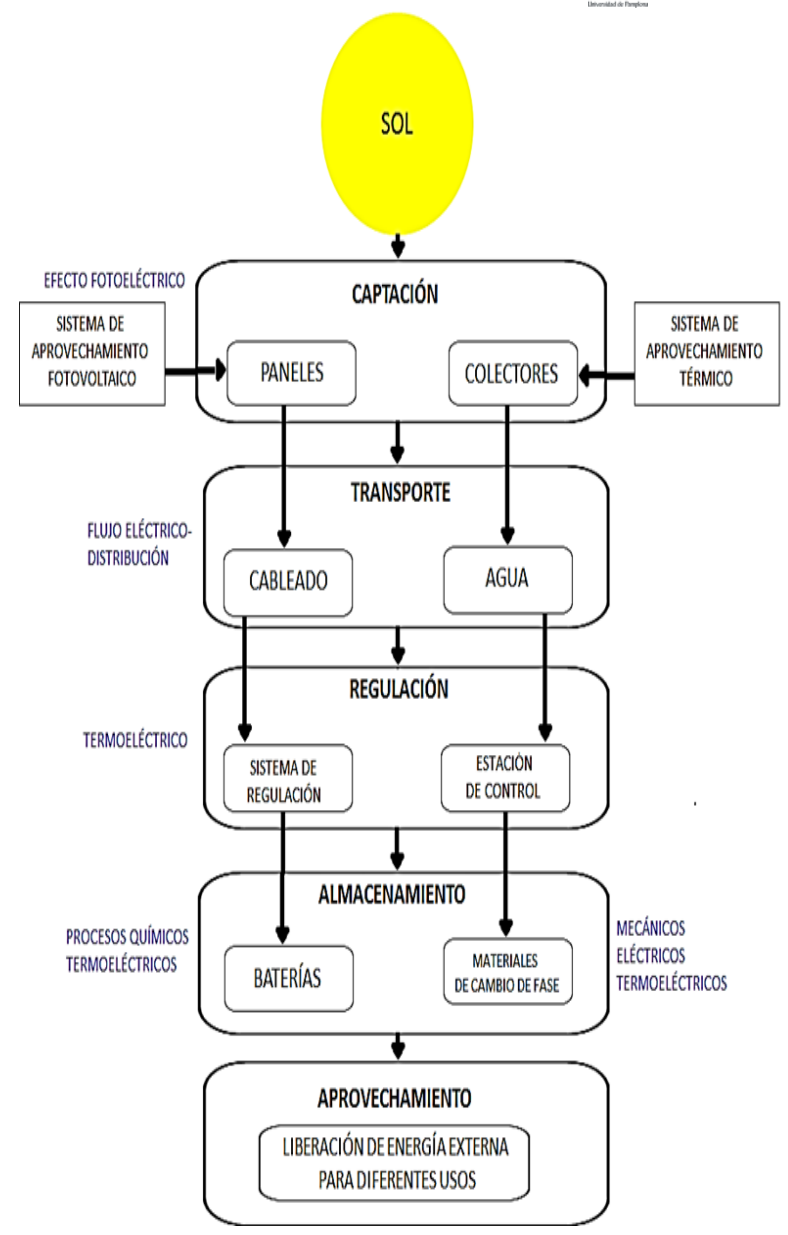

FIGURA 3. Diagrama de flujo de aprovechamiento de la energía solar en los sistemas tecnológicos-procesos 